Articlë type: Advanced Review Article title: Label-free detection and manipulation of single biological
nanoparticles

\title{
Authors:
}

Full name and affiliation; email address if corresponding author; any conflicts of interest

\author{
First author \\ Michael C. DeSantis \\ Department of Pharmaceutical Sciences, College of Pharmacy, University of Michigan, \\ Ann Arbor, MI 48109, USA
}

Second author

Wei Cheng

Department of Pharmaceutical Sciences, College of Pharmacy, University of Michigan, Ann Arbor, MI 48109, USA

Department of Biophysics, University of Michigan, Ann Arbor, MI 48109, USA

chengwe@umich.edu

\section{Third author}

[Type information here]

\section{Abstract}

In the past several years, there have been significant advances in the field of nanoparticle detection for various biological applications. Of considerable interest are synthetic nanoparticles being designed as potential drug delivery systems as well as naturally occurring or biological nanoparticles, including viruses and extracellular vesicles. Many infectious diseases and several human cancers are attributed to individual virions. Because these particles likely display different degrees of heterogeneity under normal physiological conditions, characterization of these natural nanoparticles with single-particle sensitivity is necessary for elucidating information on their basic structure and function as well as revealing novel targets for therapeutic intervention. Additionally, biodefense and point-of-care clinical testing demand ultrasensitive detection of viral pathogens particularly with high specificity. Consequently, the ability to perform label-free virus sensing has motivated the

This is the author manuscript accepted for publication and has undergone full peer review but has not been through the copyediting, typesetting, pagination and proofreading process, which may lead to differences between this version and the Version of Record. Please cite this article as doi: 10.1002/wnan.1392

This article is protected by copyright. All rights reserved. 
development of multiple electrical, mechanical, and optical-based detection techniques, some of which may even have the potential for nanoparticle sorting and multi-parametric analysis. For each technique, the challenges associated with label-free detection and measurement sensitivity are discussed as are their potential contributions for future real-world applications.

Introduction

Nanoparticle research is increasingly becoming one of the most studied fields in science as a result of the sheer diversity of nanoparticles and their potential biomedical and technological applications. As early as 1857, the unusual optical properties of nanometer-scale metals were first reported as being different compared to that of the bulk material largely owing to the high surface area to volume ratios. ${ }^{1}$ These size-dependent properties include quantum confinement in semiconductor physics, surface plasmon resonance, and superparamagnetism in various nanoparticles. Moreover, metallic nanoparticles can be modified for many diagnostic and clinical applications in medicine including fluorescent labeling, drug and gene delivery systems, and the detection and purification of biological molecules. Consequently, polymer- and liposome-based synthetic nanoparticles are now in practical use throughout the pharmaceutical industry yielding better control over size, particle dispersal and absorption, surface functionalization with proteins or other desired chemicals, and lower toxicity profiles. Nevertheless, characterization of naturally occurring (biological) nanoparticles, specifically viruses, remains of vital interest for elucidating information on basic structure and function, revealing novel targets for therapeutic intervention as well as ultrasensitive detection for biodefense; for this reason, they will be the primary focus of this review.

In addition to infectious diseases, viruses have been linked to several human cancers. The detailed mechanism of infection is not always known but can usually be attributed to a single virion. Consequently, detection and characterization of single biological nanoparticles is necessary, particularly under physiological conditions, such that solutions may contain heterogeneous populations of particles with different physical and optical properties. To unequivocally characterize natural nanoparticles, the following criteria are of importance: (1) label-free sensing, (2) high sensitivity for single nanoparticles and, if possible, multi-parametric analysis (to examine properties such as size, fluorescence, refractive index, electric charge, etc.), (3) high throughput/speed, (4) low technical difficulty (which can be achieved through automation), (5) nanoparticle heterogeneity (for a range of particle sizes, materials, and protein incorporation), (6) manipulation and/or nanoparticle sorting, and (7) preservation of the specimen's biological activity. Among these various attributes, the need for single-particle sensitivity is critical because these particles likely display significant heterogeneity even within a single population. ${ }^{2}$

In this article, we will review recent advancements for label-free detection of single, natural nanoparticles and the extent to which these characterized particles can be further manipulated for 
scientific study. Electron microscopy and other methods that usually lead to destruction of the sample are not ideal and will not be emphasized. Moreover, most fluorescence-based detection techniques, such as fluorescence resonance energy transfer (FRET) and superresolution microscopy, by themselves are not suitable as they require fluorescence labeling of the nanoparticle.

Additionally, fluorescence correlation spectroscopy (FCS) provides quantitative information typically on an ensemble of diffusing particles instead of individual particles. To focus on label-free sensing and sorting of single biological nanoparticles in this review, we have categorized the methods according to electrical-, mechanical-, and optical-based detection. For each technique, the challenges associated with label-free detection and measurement sensitivity are discussed as are their potential contributions for future real-world applications.

\section{Electrical-based detection}

Advances in nano-electronics have afforded new classes of optical biosensors incorporating photovoltaic cells, chemical, electrochemical, and photodiode elements. Traditionally, electric charge-based detection uses a local sensor to measure a change in impedance, ionic conductance, or capacitance as biomolecules transiently bind to or transit along conducting/semiconducting surfaces. Polymer and metal-based nanowires were among the first materials used in the detection of whole bacteriophages and influenza A viruses ${ }^{3,4}$ while thin film carbon nanotube (CNT)-based biosensors have been employed more recently. ${ }^{5,6}$ Here, nanowires are highly sensitive to perturbations to the local environment/nanowire surface with respect to its electrical properties resulting from either the adsorption of charged molecules or changes in ion concentration (Figure 1). Measurements include increased nanowire resistance owing to a gating effect between two contact electrodes or conductance changes when using field-effect transistors. Single-virus sensitivity has been reported. ${ }^{3}$ However, in the absence of other measurement techniques, these devices primarily provide only a high confidence level for stochastic sensing due to incubation with antibodies for selectivity, which ultimately limits what biomolecules can be detected. In addition, biomolecules in heterogeneous solutions are detected simultaneously prohibiting individual selection and subsequent manipulation. Furthermore, a decline in detection sensitivity may occur under physiological conditions where ionic concentrations are higher.

Many of these limitations are overcome using nanochannels or nanopores coupled with electrical sensing technology which permit single particle analysis and sorting. Once again, changes in conductance are measured as a nanoparticle transits through a nanopore(s) without the requirement of specific binding to antibody-coated surfaces (Figure 2). Accordingly, individual viruses can be characterized with high throughput. In one such study, the size and concentration of T7 bacteriophages in both salt solutions and blood plasma were determined as the particles passed through two voltage-biased electrodes embedded within a microfluidic channel. ${ }^{7}$ A common technique, however, is to utilize (tunable) resistive-pulse sensing in which the observed current change or blockade magnitude is proportional to the volume of electrolyte displaced by the particle. Consequently, determination of particle size and sample concentration in addition to electrophoretic 
mobility and surface charge has been performed in the case of hepatitis B virus (HBV) capsids, ${ }^{8,9}$ extracellular vesicles including microvesicles and exosomes, ${ }^{10}$ as well as a host of other biomolecules within the biomedical and pharmaceutical industries. Moreover, McMullen et al. have used a solidstate nanopore to align and transiently trap the stiff, filamentous virus $f d$ as well as measure its diffusion coefficient as a result of an electric field gradient. ${ }^{11}$ Nanopore-based detection offers relatively high speed sizing and analysis of individual nanoparticles that can also be incorporated into microfluidic platforms (i.e., 'lab-on-a-chip' or LOC devices) greatly increasing particle throughput. The high signal-to-noise ratio of resistive-pulse sensing allows for discrimination and characterization of virus-sized nanoparticles, such that for smaller particles $(<100 \mathrm{~nm})$, the salt concentration of the electrolyte can be increased or the size of the nanopore can be decreased to induce detectable current changes. However, a potential problem of this approach is the change in osmotic pressure. For biological particles, such as enveloped viruses, this unbalanced tonicity may result in morphological changes of these particles. Moreover, this approach is limited by the size of nanopores. Although smaller pores offer greater sensitivity, large particles or particulate matter/components in buffered solutions can lead to clogging of the nanopores, such that a population of smaller nanoparticles may fall out of the range of detection.

\section{Mechanical-based detection}

Arguably the most direct way to detect and/or isolate single nanoparticles is based on physical measurements. Unfortunately, as the size of the desired particle decreases so does the number of available methods which must rely on increasingly smaller nanostructures either in the form of nanofluidic channels or microcantilevers. In the first case, fabrication of planar nanochannels using photolithography to create LOC devices allows for laminar flow that can be driven by capillary action. Connecting multiple nanochannels with decreasing heights in series, injected nanoparticles become physically trapped when their sizes exceed the channel's dimensions. Fluorescence-based detection at the trapping interface has been demonstrated with polystyrene beads but also with biological materials including capsids from herpes simplex virus I (HSV-1) and HBV. ${ }^{12}$ Alternatively, combining these nanochannels as a branched network and using electrical sensors (see previous section) at several junctions permits real-time sorting based on the measured electric current and molecule length. ${ }^{13}$ While the analytes can be retrieved in the latter design after being sorted into desired outlets, only DNA molecules have been examined thus far.

Most mechanical detectors, however, utilize cantilevers that undergo deflection in response to adsorbed nanoparticles. Unlike atomic force microscopy (AFM) whose operational modes can work in liquid environments, single nanoparticle detectors typically require a vacuum for accurately measuring the frequency shifts of oscillating microcantilevers; a high mechanical quality factor (highQ), which is a measure of damping, as well as the resonator's mass primarily dictate the system's resolution. However, Lee et al. have eliminated viscous loss by using suspended nanochannel resonators (SNRs) in which fluid is placed directly inside the resonator. By fabricating nanochannels into the cantilever, SNRs have achieved a mass resolution of $2.7 \times 10^{-20} \mathrm{~kg}$ in a $1 \mathrm{kHz}$ bandwidth such 
that gold nanoparticles with diameters down to $20 \mathrm{~nm}$ can be detected. ${ }^{14}$ As a result, detection and weighing of individual nanoparticles, including viruses and protein aggregates with comparable masses, should be possible. In general, cantilever-based detectors are expensive, demand that samples are adsorbed onto substrates which forbid subsequent sorting, and are susceptible to artifacts arising from unusual sample/nanoparticle topology or from coarse probes. Nevertheless, further miniaturization can lead to increased sensitivity and broader applications in both biophysics and materials science.

\section{Optical-based detection}

For the past 400 years, optical microscopy has been the conventional technique used by scientists for visualization of larger, micron-sized particles often relying on visible light. Modern developments in charge coupled device (CCD) and CMOS cameras have afforded high spatiotemporal resolution while allowing the capture of digital images to aid in data analysis. As a result, many subfields have emerged including spectroscopy as well as absorption and fluorescence microscopy for studying nanoparticles. While fluorescence microscopy can achieve single-molecule sensitivity using superresolution methods or single particle tracking (SPT) analysis, additional labeling is normally required to visualize the biomolecule or protein of interest. In lieu of this, advancements in light scattering techniques have allowed imaging of single nanoparticles with physical sizes well below the diffraction limit. In contrast to dynamic light scattering (DLS) which monitors the time-dependent changes in the scattering intensities for an ensemble of particles in bulk suspensions, nanoparticle tracking analysis (NTA) $)^{15}$ and evanescent wave light scattering ${ }^{16}$ directly image scattered light to track the Brownian motion of an individual particle $(>10 \mathrm{~nm})$; by assuming spherical particles, the size of individual particles can be derived from the measured diffusion coefficients based on the Stokes-Einstein equation. The NTA technique has been commercialized and can perform label-free measurements of nanoparticles and protein aggregates in suspension. However, there remain challenges associated with the reproducibility and peak identification of the calculated size distributions. Moreover, photothermal heterodyne imaging of virus-like nanoparticles with various absorption properties ${ }^{17}$ and far-field emission of photonic molecule-conjugated nanoparticles ${ }^{18}$ have been demonstrated. Here, we focus on several traditional optics-based methods that have recently characterized viruses, some of which are capable of nanoparticle sorting.

\section{Refractive-index based detection}

The refractive index (RI), defined as the ratio between the speed of light in vacuum and its phase velocity in the material of interest, is a fundamental optical property of materials. Consequently, it potentially offers a label-free parameter for distinguishing materials of different compositions.

Because the transient binding or immobilization of biomolecules at an interface alters the local RI, there are corresponding changes to the measured optical signals which are subsequently used to detect these events. Various structures include surfaces composed of thin metal layers or metallic nanoparticles for surface plasmon resonance (SPR), photonic crystals (PCs), optical ring resonators or 
other nanocavities to achieve light energy confinement; several of these structures have already been incorporated into microfluidic channels to increase throughput and further minimize solution volumes. In the context of biological nanoparticles, we shall discuss two of these techniques in greater detail.

\section{Surface plasmon resonance}

To measure this change in the local RI, one form of implementation among many others, are devices that utilize SPR. In a typical setup, a thin layer of metal (the interface, usually gold) is sandwiched between glass and aqueous media. A laser beam is projected to the back of the metal layer under total internal reflection conditions to create a field of evanescent waves. When the angle of laser incidence equals the so-called 'resonance angle', the electron clouds ('plasmon') in the metal will absorb photon energy from the evanescent field, and the resulting loss of light intensity can be detected by measuring the reflected laser beam. This resonance angle depends on the local RI, which changes in response to molecules binding at the interface. Therefore, by measuring the angle of laser reflection, laser intensity changes at a fixed wavelength, or wavelength shifts in the light spectrum, one can measure the binding of molecules in real time. The sensitivity of RI-based sensors that involve resonant modes is often reported as a ratio of the magnitude of the spectral shift to the corresponding RI change, the latter being measured in refractive index units (RIUs). In comparison to other methods, SPR can achieve higher detection sensitivities of $>10000 \mathrm{~nm} / \mathrm{RIU}$, depending on the resonance wavelength used. ${ }^{19}$ Also important is the system's resolution which denotes the smallest spectral shift that can be accurately measured. Together, these quantities determine the detection limit quantifying the minimum amount of adsorbed protein the sensor can measure. While SPR has been used extensively to study molecular adsorption of polymers, DNA, and proteins, detection of single nanoparticles, including viruses and exosomes, has only recently been demonstrated. In 2010, Wang et al. measured the intensities of both human cytomegalovirus (HCMV) and H1N1 influenza viruses alongside silica nanoparticles (with comparable size, $d=98 \mathrm{~nm}$ and $\mathrm{RI}, n=1.46$ ), on surfaces functionalized with and without antibodies yielding size and mass distributions with a detection limit of $0.2 \mathrm{fg} / \mathrm{mm}^{2}$ (Figure 3). ${ }^{20}$ Nanoplasmonic-based detection has also been used to analyze intact human immunodeficiency viruses (HIV) of varying subtypes selectively captured on antibody immobilized biosensing surfaces at clinically relevant concentrations from unprocessed whole blood. ${ }^{21}$ For the case of exosomes (50-100 nm in diameter), Im et al. recently built an SPR-based sensor called the nano-plasmonic exosome (nPLEX) assay consisting of a series of nanohole arrays coated with specific antibodies for profiling surface-exposed proteins to analyze exosomes derived from ovarian cancer cells; ${ }^{22}$ similar assays have also been developed. ${ }^{23,}{ }^{24}$ SPR-based detection techniques can achieve single-particle sensitivity and provide a low-cost, high throughput design for biomolecule analysis, especially for point-of-care (POC) medical testing, independent of solution conditions

Optical ring resonators

This article is protected by copyright. All rights reserved. 
Similar in idea to the above SPR devices, the local RI change due to binding of molecules at the interface can also be measured in a dielectric microparticle, or the so-called "resonant microcavity". ${ }^{25}$ At the resonant wavelength, light coupled to the cavity via an input waveguide will increase in intensity due to constructive interference as it cycles within the structure before detection at an output bus waveguide; optical coupling is affected by the distance, the coupling length, and the difference in RIs. As a result, whispering gallery modes (WGMs) are formed within the cavity such that its evanescent field can be locally perturbed by binding of molecules at the particle surface, which causes a measurable shift in the resonance frequency. Early studies demonstrated the sensitivity of optical ring resonators as a biosensor by measuring the binding of proteins, such as biotin, on the microparticle surface; in addition to bulk RI detection, the corresponding noise equivalent detection limit of $0.14 \mathrm{pg} / \mathrm{mm}^{2}$ had been reported. ${ }^{25,26}$ Subsequent work with influenza A viruses has demonstrated sensitivity for detection of a single virus in aqueous buffer. The small size of the influenza A virus demanded reduction in the microcavity size to amplify the magnitude shift of the resonance frequency (Figure 4). ${ }^{27}$ Similar work has been done for bean pod mottle viruses. ${ }^{28}$ While various environmental noise factors can distort measurements based on resonance frequency shifts, the temporal resolution of WGMs is on par with photon lifetimes, $\tau=$ $Q / \omega \approx 10^{-10}$ s despite lower $Q$ values associated with smaller resonator cavities having increased energy leakage. To improve optical coupling since the signal amplitude is sensitive to the location of nanoparticle binding with respect to the evanescent tail of the WGM, it is recommended that the WGM shifts, broadenings, and splittings be monitored as well. Consequently, Zhu et al. utilized split WGMs in an ultrahigh- $Q$ resonator to analyze the size distributions $(d=106.4 \pm 11.0 \mathrm{~nm})$ and polarizabilities of single influenza A virions. ${ }^{29}$ They estimated that the detection limit for dielectric nanoparticles $(n=1.5)$ is approximately $20 \mathrm{~nm}$ such that the smallest polystyrene particles accurately measured were $60 \mathrm{~nm}$ in diameter. Moreover, a reference interferometer operating in conjunction with an ultrahigh-Q microcavity offers significantly increased sensitivity to resolve frequency shifts in the detection of influenza A virions. ${ }^{30}$ Optical resonators can easily be adapted for use on microfluidic or LOC devices requiring little mode volume to accurately characterize individual nanoparticles, although a significant weakness concerns the sequential binding of particles to the resonator surface which can change WGM characteristics over time. In summary, both SPR and WGM techniques are sensitive for detection down to single particles and confer high selectivity because biomolecules require antibody-coated surfaces for binding. As a result, further manipulation of these detected particles is prevented.

\section{Interferometry}

Unlike absorption microscopy which requires that nanoparticles have intrinsic absorption properties making them suitable for study, interferometric methods rely on the scattered intensities of particles illuminated by coherent light. While direct imaging of non-fluorescent nanoparticles is possible, as discussed above, the signal intensities are typically weak for smaller particles owing to an $r^{6}$ dependence thus greatly reducing detection sensitivity. Consequently, interferometry allows 
resolution enhancement of optical microscopy by numerical combining of partial images with registered amplitude as well as phase. Based on the frequencies of the initial waves, the resulting interference pattern provides measurements of small displacements, RI changes, and surface irregularities within the examined sample. One of the earliest techniques involved wavefront splitting interferometers to observe the phase difference of light passing through an aperture yielding constructive or destructive interference. Accordingly, HSV-1 virions bound to antibodycoated waveguide surfaces have been detected using a Young interferometer. ${ }^{31}$ As a detector only, direct measurements, including size estimation of the nanoparticles, are not performed. While the analyte concentration can be determined, the detection limit may not be sensitive to single particles. A common method to increase sensitivity is by non-linear mixing of scattered light with a reference field through homodyne detection in which the two beams are of the same wavelength. Heterodyne detection uses a stronger reference beam to amplify the scattered field intensity and shift it to a new frequency range as either the sum or difference of the respective frequencies; the new signal will have an intensity proportional to the product of the amplitudes of the two input signals. The latter has been used notably by Mitra et al. for real-time detection and characterization of several viruses including influenza, Sindbis, and HIV, within nanofluidic channels (Figure 5). ${ }^{32-34}$ Single-virus sensitivity has been demonstrated. ${ }^{32}$ To further improve the detection signal via phase imaging, Daaboul et al. developed an interferometric reflectance imaging sensor (IRIS) so as to immobilize nanoparticles, including influenza H1N1 and vesicular stomatitis virus (VSV)-pseudotyped virions, on a reflective $\mathrm{Si} / \mathrm{SO}_{2}$ substrate. ${ }^{35,36}$ Briefly, a top oxide layer minimizes the optical path difference at specific wavelengths resulting in closely aligned scattered and reference fields. The enhanced interferometric response of nanoparticles on the surface can then be imaged at different wavelengths for detection of individual particles. For these techniques, prior knowledge about the virus, such as its $\mathrm{RI}$ and behavior as non-adsorbing, spherical Rayleigh scatterers as well as experimental parameters used in the latter's model, is required for accurate sizing.

\section{Optical trapping}

Invented by A. Ashkin, ${ }^{37}$ optical tweezers (OTs) have emerged as a powerful tool in biophysics for trapping and manipulation of nanometer- and micron-sized particles. For typical single-beam gradient OTs, they are constructed by focusing a laser beam to a diffraction-limited spot using an objective lens with a high numerical aperture. At the narrowest point of a tightly focused laser beam, radiative pressure due to the transfer of momentum by incident photons will cause a dielectric particle to experience a scattering and gradient force. As the name implies, the scattering force will tend to push the particle in the direction of light propagation whereas the gradient force, proportional to the dipole moment of the dielectric, will pull the particle towards the focal region. If the axial component of the gradient force is greater than that of the scattering force, a particle is stably trapped in three dimensions such that its axial equilibrium position is located slightly beyond the focus. Generally, OTs use continuous-wave (cw) lasers operating in the infrared or NIR regime. This is advantageous for biological specimens because they have less absorption in the NIR window 
where light has its maximum depth of penetration in tissue. Nevertheless, one should pay attention to the wavelengths selected as potential photodamage to trapped particles can occur. For example, molecular oxygen can absorb around $1064 \mathrm{~nm}$ and generate reactive oxygen species in the presence of a sensitizer. ${ }^{38}$ To avoid this photodamage, an $830 \mathrm{~nm}$ laser is strongly preferred which also reduces heating effects due to water absorption. ${ }^{39,} 40$ Although primarily used for trapping, NIR cw lasers are also capable of simultaneous two-photon excitation of fluorescent proteins in optical traps or inside eukaryotic cells with single-molecule sensitivity. ${ }^{41,42}$

Compared to micron-sized objects and bacterial or mammalian cells, the trapping of biological nanoparticles is complicated by their small size and poorly characterized RIs. While it was previously demonstrated that larger sized viruses, such as the tobacco mosaic virus (TMV), and bacteria could be easily trapped, most animal viruses are typically less than $300 \mathrm{~nm}$ in size. ${ }^{43}$ Because the gradient force scales as $r^{3}$, the photon forces will be considerably weaker for these particles compared to typical micron-sized objects; thus, particles may have a higher tendency to escape after being trapped. To confirm the trapping of these individual particles, it is thus necessary to provide additional measurements on the trapped particles to show that these are indeed single particles as expected.

Using a single-beam gradient optical trap in combination with confocal microscopy, Bendix and Oddershede has successfully demonstrated optical trapping of unilamellar lipid vesicles as small as $50 \mathrm{~nm}$ that encapsulate high concentrations of sucrose solutions. ${ }^{44}$ By combining an optical trap and single-molecule two-photon fluorescence (TPF) detection, Pang et al. has demonstrated optical trapping of individual HIV-1 virions in culture media (Figure 6). ${ }^{2}$ Because the TPF is excited by the trapping laser right at the focus and measurable at the single-molecule level owing to the ultra-small excitation volume, this technique is well suited to detect and characterize nanoscale, dielectric particles with single-molecule resolution. They have thus named this technique 'virometry', following 'cytometry', the powerful technique that has been widely used to characterize heterogeneity among individual cells. An additional advantage of this technique is the use of backfocal-plane interferometry (BFPI) ${ }^{45}$ which permits ultra-sensitive tracking of the damped Brownian motion of the trapped particle at high bandwidth $(62.5 \mathrm{kHz})$. Fitting of the corresponding power spectrum yields the diffusion coefficient and corner frequency of the trapped particle with high precision. ${ }^{46}$ It was observed that these parameters are highly sensitive to the simultaneous trapping of multiple particles such that individual virions can be easily distinguished from the total population. By using an independent displacement reference, BFPI offers precision measurement of the diameters of the trapped particles and can thus be used to directly quantitate the heterogeneity among particles based on their size. Combined with simultaneous TPF detection using either external or internal fluorescent labels, virometry truly offers multi-parametric analysis for an individually trapped particle and is well suited for measuring heterogeneity of nanoparticles in solution with high sensitivity. The single-molecule resolution offered by TPF is particularly important for measurement of biological viruses because these particles are likely to be highly heterogeneous 
with respect to their protein content. One example is the density of envelope glycoproteins on the surface of individual HIV-1 virions. Previous cryoelectron microscopy studies of individual HIV-1 virions derived from chronically infected T cell lines indicated a broad heterogeneity in the density of these proteins per virion, ranging from 4 to $35 .{ }^{47}$ Using optical trapping virometry, Pang et al. showed that the density of these proteins can vary by over one order in magnitude for individual HIV-1 virions derived from transfected cell lines. Given the apparent low copy number of this protein and the large variation across individual virions, single-molecule sensitivity is therefore essential to reveal these heterogeneities. Although single-molecule fluorescence detection has become routine nowadays in many labs, for example, through the implementation of total internal reflection fluorescence (TIRF) microscopy to study the dynamics of HIV-1 envelope glycoproteins, ${ }^{48}$ virometry is the only technique available that offers multi-parametric analysis for individual, nanoscale particles in solution with single-molecule sensitivity. As a result, individual virions can be characterized in real time and manipulated. This capability also makes it possible to directly deliver a single virion to a host cell for studying the early events in viral infection. Additionally, the unprecedented level of control in manipulating virus-like particles serves as motivation to incorporate OTs in microfluidic platforms, possibly in combination with other optical-based techniques, to achieve high-throughput nanoparticle detection.

Fabrication of optical nanostructures has also paved the way for near-field optical trapping including holographic and plasmonic OTs that are capable of 3D manipulation of nanoscale, dielectric objects. ${ }^{49}$ Using a photonic crystal resonator embedded within a microfluidic chamber to create a highly localized optical cavity, Kang et al. has recently demonstrated optical trapping of individual influenza H1N1 virions in free solution..$^{50}$ In order to estimate the stoichiometry of bound antibodies to a single virion, they have developed an effective sphere model relating the change in trap stiffness and RI after incubation with specific antibodies. In this approach, evanescent wave light scattering produces an image of the particle from which 2D localization measurements are made. The resulting positional variance is used to calculate the trap stiffness with the equipartition theorem. The stoichiometry of bound antibodies per virion can then be estimated based on this quantitative model. This technique has the potential to be exquisitely sensitive to biomolecular binding in free solutions and provides binding parameters in a label-free manner.

\section{Flow cytometry}

Flow cytometers are ubiquitous throughout the biotechnology and pharmaceutical industries as they serve as powerful tools in the identification and analysis of heterogeneous samples of cell populations. Historically, most commercial instruments were designed for rapid counting/sorting of cells and similarly sized particles in real time; consequently, they are routinely used diagnostically in the medical community, especially for blood cancer screening. Detection is initially based on forward scattered (FSC) and side-scattered (SSC) light which convey an object's size as it passes through a stationary laser focus; however, multi-parametric analysis is usually performed by including 
additional lasers and photomultipier tubes (PMTs) for excitation and fluorescence detection, respectively. To ensure that particles or cells are in single-file passage through the observation region, the sample is typically delivered by hydrodynamic focusing using sheath fluid, or through a microcapillary, or a combination of hydrodynamic focusing plus acoustic focusing. Here, analysis and sorting of heterogenous mixtures can be achieved through fluorescence-activated cell sorting (FACS). Briefly, a vibrating mechanism causes the stream to break into individual droplets containing no more than one cell. At the same time, an electrical charging ring places a charge on the droplet according to a fluorescence measurement taken previously with respect to a parameter of interest. Finally, an electrostatic deflection system diverts the droplet into an appropriate container based on its charge. ${ }^{51}$ Most flow cytometers on the market can detect particles with diameters larger than 300 $\mathrm{nm}$. For particles much smaller than the wavelength of the light, the so-called Rayleigh scatterers, the scattered light varies as $r^{6}$ which makes it complicated to detect these particles with sufficient signal-to-noise ratios above background. With significant modification, flow cytometers have been used to analyze nanoparticles; however, proper discrimination of single particles $(<200 \mathrm{~nm}$ ) from doublets or aggregates, based on FSC signals alone, is a major challenge. Indeed, studies using fluorescently-labeled Junin viruses (JUNV) yielded FSC and fluorescent signals that mostly lie at the background noise level and therefore indistinguishable from the bulk suspension. ${ }^{52}$ To overcome this hurdle, researchers can rely on brighter signals from fluorescently-labeled particles, including cellderived vesicles $(d \approx 100 \mathrm{~nm}),{ }^{53}$ or conjugate magnetic nanoparticles to virions as in the case of HIV-1 to perform so-called "flow virometry". ${ }^{54}$ Thus, it is very difficult to achieve label-free sensing of individual nanoparticles while preserving the biological activity/infectiousness of viruses.

Alternatively, Zhu et al. has developed a highly sensitive flow cytometry that allows detection of single silica and gold nanoparticles as small as 24 and $7 \mathrm{~nm}$ in diameter, respectively, based on light scattering. ${ }^{55}$ This work is made possible by confining the sample to a narrow sheath flow and isolation of the detection region far away from the walls of the flow cell so as to reduce background scattered light. With this development, they have also analyzed doxorubicin-carrying liposomes $(d=$ $61 \pm 8 \mathrm{~nm}$ ) and siRNA-loaded lipid nanoparticles. However, it remains to be demonstrated if this technique will work for extracellular vesicles or viruses that have comparatively low RIs.

\section{Conclusion}

The past several years have seen tremendous advances in this fast-growing field of nanoparticle detection for various biological applications. This is important for sensing of viruses as well as characterization of viral pathogens and drug delivery nanoparticles, ${ }^{56}$ just to name a few. Many techniques with single-nanoparticle sensitivity have been developed and each technique has its own strengths and weaknesses. In the future, it is likely that different techniques will be used in different settings for the specific application to be addressed. For example, RI-based measurements such as SPR and optical ring resonators are especially well suited for POC applications. They require specific antibodies for capture of the nanoparticles but are highly sensitive, low-cost, and potentially high throughput. For quality control of drug delivery nanoparticles, high throughput is almost essential. In this regard, additional modifications of existing flow cytometry techniques to increase detection 
sensitivity for nanoparticles are likely to be made. This development is much desired given the multiparametric analysis capability that is already built-in among many commercial models. A potential bottleneck along this development in flow cytometry, however, is the sensitivity for fluorescence detection. To measure the heterogeneity among nanoparticles, especially for biological particles such as viruses that may display broad heterogeneity, single-molecule detection sensitivity is highly desired. Although this problem can be alleviated by engineering brighter fluorophores, this bottleneck resides partly in the fluorescence detection scheme. To achieve single-molecule sensitivity for particles in fluid, reduction of the background fluorescence is essential; consequently, confocal or multi-photon excitation ${ }^{2}$ is very effective. However, due to the reduced excitation volume, in order to quantitate nanoparticle heterogeneity precisely, one has to guarantee that the nanoparticle will travel through the laser focus with minimal deviation. Whether this is feasible with existing sheath fluid techniques remains to be seen. Alternatively, other ideas may be tested such as finer control to trap single particles in solution using hydrodynamic flow ${ }^{57}$ or through a feedback control mechanism to apply corrective voltages in the anti-Brownian electrokinetic (ABEL) trap. ${ }^{58}$

Many optical-based detection techniques for nanoparticles involve measurement of scattered light from the nanoparticles of interest. One should be cautioned that the scattered light from the nanoparticle will be a function of both the particle's size and RI. These two parameters are linked for quantitative interpretation of the scattered light. Due to the lack of information on the RI of biological nanoparticles, ${ }^{59}$ many studies in the field have adopted a convention to assume a RI for the particle of interest which has not been validated by experimental measurements. Because uncertainties in the particle's RI directly propagate into the uncertainty of the particle's size, a reliable measurement of the RI for these particles will be essential for accurate data analysis. Thus, this will be an important area for future study.

Lastly, as have been seen for several of the techniques that are capable of single-virus detection, their success also relies on the rational design of a microfluidic or nanofluidic device. These devices might be critical for measurement of nanoparticles with high sensitivity and throughput as well as the potential for manipulation and sorting of these particles. Accordingly, development of these devices in parallel for nanoparticle detection is also envisioned.

References

1. Faraday M. The Bakerian Lecture: Experimental relations of gold (and other metals) to light. Philos Trans R Soc Lond 1857, 147:145-181.

2. Pang Y, Song H, Kim JH, Hou X, Cheng W. Optical trapping of individual human immunodeficiency viruses in culture fluid reveals heterogeneity with single-molecule resolution. Nat Nanotechnol 2014, 9:624-630.

3. Patolsky F, Zheng G, Hayden O, Lakadamyali M, Zhuang X, Lieber CM. Electrical detection of single viruses. Proc Natl Acad Sci U S A 2004, 101:14017-14022. 
4. Shirale DJ, Bangar MA, Park M, Yates MV, Chen W, Myung NV, Mulchandani A. Label-free chemiresistive immunosensors for viruses. Environ Sci Technol 2010, 44:9030-9035.

5. Garcia-Aljaro C, Cella LN, Shirale DJ, Park M, Munoz FJ, Yates MV, Mulchandani A. Carbon nanotubes-based chemiresistive biosensors for detection of microorganisms. Biosens Bioelectron 2010, 26:1437-1441.

6. Mandal HS, Su Z, Ward A, Tang XS. Carbon nanotube thin film biosensors for sensitive and reproducible whole virus detection. Theranostics 2012, 2:251-257.

7. Fraikin JL, Teesalu T, McKenney CM, Ruoslahti E, Cleland AN. A high-throughput label-free nanoparticle analyser. Nat Nanotechnol 2011, 6:308-313.

8. Zhou K, Li L, Tan Z, Zlotnick A, Jacobson SC. Characterization of hepatitis B virus capsids by resistive-pulse sensing. J Am Chem Soc 2011, 133:1618-1621.

9. Harms ZD, Haywood DG, Kneller AR, Selzer L, Zlotnick A, Jacobson SC. Single-particle electrophoresis in nanochannels. Anal Chem 2015, 87:699-705.

10. Maas SL, De Vrij J, Broekman ML. Quantification and size-profiling of extracellular vesicles using tunable resistive pulse sensing. J Vis Exp 2014:e51623.

11. McMullen A, de Haan HW, Tang JX, Stein D. Stiff filamentous virus translocations through solid-state nanopores. Nat Commun 2014, 5:4171.

12. Hamblin MN, Xuan J, Maynes D, Tolley HD, Belnap DM, Woolley AT, Lee ML, Hawkins AR. Selective trapping and concentration of nanoparticles and viruses in dual-height nanofluidic channels. Lab Chip 2010, 10:173-178.

13. Yamamoto T, Fujii T. Nanofluidic single-molecule sorting of DNA: a new concept in separation and analysis of biomolecules towards ultimate level performance. Nanotechnology 2010, 21:395502.

14. Lee J, Shen W, Payer K, Burg TP, Manalis SR. Toward attogram mass measurements in solution with suspended nanochannel resonators. Nano Lett 2010, 10:2537-2542.

15. Filipe V, Hawe A, Jiskoot W. Critical evaluation of Nanoparticle Tracking Analysis (NTA) by NanoSight for the measurement of nanoparticles and protein aggregates. Pharm Res 2010, 27:796-810.

16. Matsuoka H. Evanescent wave light scattering - A fusion of the evanescent wave and light scattering techniques to the study of colloids and polymers near the interface. Macromol Rapid 2001, 22:51-67.

17. Vieweger M, Goicochea N, Koh ES, Dragnea B. Photothermal imaging and measurement of protein shell stoichiometry of single HIV-1 Gag virus-like nanoparticles. ACS Nano 2011, 5:7324-7333.

18. Zhang N, Liu S, Wang K, Gu Z, Li M, Yi N, Xiao S, Song Q. Single nanoparticle detection using far-field emission of photonic molecule around the exceptional point. Sci Rep 2015, 5:11912.

19. Pang L, Hwang GM, Slutsky B, Fainman Y. Spectral sensitivity of two-dimensional nanohole array surface plasmon polariton resonance sensor. Appl Phys Lett 2007, 91:123112.

20. Wang S, Shan X, Patel U, Huang X, Lu J, Li J, Tao N. Label-free imaging, detection, and mass measurement of single viruses by surface plasmon resonance. Proc Natl Acad Sci U S A 2010, 107:16028-16032.

21. Inci F, Tokel O, Wang S, Gurkan UA, Tasoglu S, Kuritzkes DR, Demirci U. Nanoplasmonic quantitative detection of intact viruses from unprocessed whole blood. ACS Nano 2013, 7:4733-4745. 
22. Im H, Shao H, Park YI, Peterson VM, Castro CM, Weissleder R, Lee H. Label-free detection and molecular profiling of exosomes with a nano-plasmonic sensor. Nat Biotechnol 2014, 32:490-495.

23. Zhu L, Wang K, Cui J, Liu H, Bu X, Ma H, Wang W, Gong H, Lausted C, Hood L, et al. Label-free quantitative detection of tumor-derived exosomes through surface plasmon resonance imaging. Anal Chem 2014, 86:8857-8864.

24. Grasso L, Wyss R, Weidenauer L, Thampi A, Demurtas D, Prudent M, Lion N, Vogel H. Molecular screening of cancer-derived exosomes by surface plasmon resonance spectroscopy. Anal Bioanal Chem 2015, 407:5425-5432.

25. Vollmer F, Braun D, Libchaber A, Khoshsima M, Teraoka I, Arnold S. Protein detection by optical shift of a resonant microcavity. Appl Phys Lett 2002, 80:4057.

26. Li H, Fan X. Characterization of sensing capability of optofluidic ring resonator biosensors. Appl Phys Lett 2010, 97:011105.

27. Vollmer F, Arnold S, Keng D. Single virus detection from the reactive shift of a whisperinggallery mode. Proc Natl Acad Sci U S A 2008, 105:20701-20704.

28. McClellan MS, Domier LL, Bailey RC. Label-free virus detection using silicon photonic microring resonators. Biosens Bioelectron 2012, 31:388-392.

29. Zhu J, Ozdemir SK, He L, Chen DR, Yang L. Single virus and nanoparticle size spectrometry by whispering-gallery-mode microcavities. Opt Express 2011, 19:16195-16206.

30. Lu T, Lee H, Chen T, Herchak S, Kim J-H, Fraser SE, Flagan RC, Vahala K. High sensitivity nanoparticle detection using optical microcavities. Proc Natl Acad Sci U S A 2011, 108:59765979.

31. Ymeti A, Greve J, Lambeck PV, Wink T, van Hovell SW, Beumer TA, Wijn RR, Heideman RG, Subramaniam V, Kanger JS. Fast, ultrasensitive virus detection using a Young interferometer sensor. Nano Lett 2007, 7:394-397.

32. Mitra A, Deutsch B, Ignatovich F, Dykes C, Novotny L. Nano-optofluidic detection of single viruses and nanoparticles. ACS Nano 2010, 4:1305-1312.

33. Mitra A, Ignatovich F, Novotny L. Real-time optical detection of single human and bacterial viruses based on dark-field interferometry. Biosens Bioelectron 2012, 31:499-504.

34. Block O, Mitra A, Novotny L, Dykes C. A rapid label-free method for quantitation of human immunodeficiency virus type-1 particles by nanospectroscopy. J Virol Methods 2012, 182:7075.

35. Daaboul GG, Yurt A, Zhang X, Hwang GM, Goldberg BB, Unlu MS. High-throughput detection and sizing of individual low-index nanoparticles and viruses for pathogen identification. Nano Lett 2010, 10:4727-4731.

36. Daaboul GG, Lopez CA, Chinnala J, Goldberg BB, Connor JH, Unlu MS. Digital sensing and sizing of vesicular stomatitis virus pseudotypes in complex media: a model for Ebola and Marburg detection. ACS Nano 2014, 8:6047-6055.

37. Ashkin A. Acceleration and trapping of particles by radiation pressure. Phys Rev Lett 1970, 24:156-159.

38. Landry MP, McCall PM, Qi Z, Chemla YR. Characterization of photoactivated singlet oxygen damage in single-molecule optical trap experiments. Biophys J 2009, 97:2128-2136.

39. Neuman KC, Block SM. Optical trapping. Rev Sci Instrum 2004, 75:2787-2809.

40. Cheng W, Hou X, Ye F. Use of tapered amplifier diode laser for biological-friendly highresolution optical trapping. Opt Lett 2010, 35:2988-2990. 
41. Hou X, Cheng W. Single-molecule detection using continuous wave excitation of two-photon fluorescence. Opt Lett 2011, 36:3185-3187.

42. Hou X, Cheng W. Detection of single fluorescent proteins inside eukaryotic cells using twophoton fluorescence. Biomed Opt Express 2012, 3:1047-1049.

43. Ashkin A, Dziedzic JM. Optical trapping and manipulation of viruses and bacteria. Science 1987, 235:1517-1520.

44. Bendix PM, Oddershede LB. Expanding the optical trapping range of lipid vesicles to the nanoscale. Nano Lett 2011, 11:5431-5437.

45. Gittes F, Schmidt CF. Interference model for back-focal-plane displacement detection in optical tweezers. Opt Lett 1998, 23:7-9.

46. Tolić-Nørrelykke SF, Schäffer E, Howard J, Pavone FS, Jülicher F, Flyvbjerg H. Calibration of - optical tweezers with positional detection in the back focal plane. Rev Sci Instrum 2006, 77:103101.

47. Zhu P, Liu J, Bess J, Jr., Chertova E, Lifson JD, Grise H, Ofek GA, Taylor KA, Roux KH. Distribution and three-dimensional structure of AIDS virus envelope spikes. Nature 2006, 441:847-852.

48. Munro JB, Gorman J, Ma X, Zhou Z, Arthos J, Burton DR, Koff WC, Courter JR, Smith AB, 3rd, Kwong PD, et al. Conformational dynamics of single HIV-1 envelope trimers on the surface of native virions. Science 2014, 346:759-763.

49. Berthelot J, Acimovic SS, Juan ML, Kreuzer MP, Renger J, Quidant R. Three-dimensional manipulation with scanning near-field optical nanotweezers. Nat Nanotechnol 2014, 9:295299.

50. Kang P, Schein P, Serey X, O'Dell D, Erickson D. Nanophotonic detection of freely interacting molecules on a single influenza virus. Sci Rep 2015, 5:12087.

51. Fulwyler MJ. Electronic separation of biological cells by volume. Science 1965, 150:910-911.

52. Gaudin R, Barteneva NS. Sorting of small infectious virus particles by flow virometry reveals distinct infectivity profiles. Nat Commun 2015, 6:6022.

53. Van der Vlist EJ, Nolte-'t Hoen EN, Stoorvogel W, Arkesteijn GJ, Wauben MH. Fluorescent labeling of nano-sized vesicles released by cells and subsequent quantitative and qualitative analysis by high-resolution flow cytometry. Nat Protoc 2012, 7:1311-1326.

54. Arakelyan A, Fitzgerald W, Margolis L, Grivel JC. Nanoparticle-based flow virometry for the analysis of individual virions. J Clin Invest 2013, 123:3716-3727.

55. Zhu S, Ma L, Wang S, Chen C, Zhang W, Yang L, Hang W, Nolan JP, Wu L, Yan X. Lightscattering detection below the level of single fluorescent molecules for high-resolution characterization of functional nanoparticles. ACS Nano 2014, 8:10998-11006.

56. Cressey D. Tiny traits cause big headaches. Nature 2010, 467:264-265.

57. Tanyeri M, Schroeder CM. Manipulation and confinement of single particles using fluid flow. Nano Lett 2013, 13:2357-2364.

58. Cohen AE, Moerner WE. Suppressing Brownian motion of individual biomolecules in solution. Proc Natl Acad Sci U S A 2006, 103:4362-4365.

59. van der Pol E, Coumans FA, Sturk A, Nieuwland R, van Leeuwen TG. Refractive index determination of nanoparticles in suspension using nanoparticle tracking analysis. Nano Lett 2014, 14:6195-6201.

Figure captions

This article is protected by copyright. All rights reserved. 
Figure 1 Nanowire-based detection of single influenza A viruses. (Left) Two nanowires incubated with different antibodies to promote specific binding. (Right) The corresponding changes in conductance reflects the surface charge of a virus that binds and unbinds to nanowire 2. (Reprinted with permission from Ref 3. Copyright 2004 National Academy of Sciences of the United States of America)

Figure 2 Nanochannel design for resistive-pulse sensing of HBV capsids. (a) Schematic of the microfluidic chamber with two channels for delivery of biological nanoparticles. (b) Scanning electron microscope (SEM) image of the two microchannels with an enlarged view of a $2.5-\mu \mathrm{m}$ long pore-to-pore channel. (c) AFM image of this channel incorporating pores that are $45 \mathrm{~nm}$ wide, $45 \mathrm{~nm}$ deep, and $430 \mathrm{~nm}$ long. (Reprinted with permission from Ref 9. Copyright 2014 American Chemical Society)

Figure 3 SPR microscopy for detection of influenza A viruses. (a) Schematic of the experimental setup. (b) Images of H1N1 influenza A viruses and silica nanoparticles of varying size in PBS buffer. (Insets) Corresponding nanoparticle images produced by numerical simulation. (Reprinted with permission from Ref 20. Copyright 2010 National Academy of Sciences of the United States of America)

Figure 4 Detection of influenza A virions by a microsphere resonator. (a) Illustration of a silica microsphere immersed in aqueous solution. A tunable distributed feedback laser excites WGMs of a microsphere by evanescent coupling to a tapered optical fiber. (Inset) Typical transmission spectrum for a WGM mode detected while the laser wavelength is tuned. (b) The resonance wavelength shifts associated with the binding of single influenza A virions to a microsphere cavity $(r=39 \mathrm{~mm})$ in PBS buffer. (Reprinted with permission from Ref 27. Copyright 2008 National Academy of Sciences of the United States of America)

Figure 5 Schematic of the dark-field heterodyne interferometric detection technique. Nanoparticles inside a glass nanofluidic channel are detected as they traverse an illumination spot formed using an excitation laser $\left(\mathrm{E}_{\mathrm{exc}}\right)$. The detector signal is proportional to the product of the nanoparticle's scattering field $\left(E_{s}\right)$ and a additional frequency-shifted reference field $\left(E_{r}\right)$. (Inset) SEM image of the nanofluidic channels. Scale bar $=2 \mu \mathrm{m}$. (Reprinted with permission from Ref 33 . Copyright 2012 Elsevier)

Figure 6 Optical trapping virometry of HIV-1 virions. (a) Experimental schematic for optical trapping of single HIV-1 virions in culture fluid. HIV-1 virions were delivered into a microfluidic chamber and trapped by the IR laser focused at the center of the chamber. The $x y z$ dimensions are shown as indicated, with $y$ perpendicular to the figure plane. (b) Representative TPF time courses from individually-trapped HIV-1 virions. All traces were fit with a single exponential function (red), with time constants for each trace as follows: 60.7 (square), 49.5 (cross), 51.5 (circle), and $54.1 \mathrm{~s}$ (triangle). Time zero started with the onset of TPF collection. (c) The laser deflection signal measured in real time using BFPI, which can be used to distinguish single HIV-1 particles from aggregates in 
complete media. (d) Representative Alexa-594 TPF time course from a single virion bound with monoclonal antibody b12 labeled with Alexa-594, where individual photobleaching steps are indicated with arrows. (Inset) A cartoon of a HIV-1 virion with a single envelope glycoprotein trimer. (Adapted from Ref 2. Copyright 2014 Nature Publishing Group)

\section{Further Reading/Resources}

Fan X, White IM. Optofluidic microsystems for chemical and biological analysis. Nat Photonics 2011, 5:591-597.

Ko J, Carpenter E, Issadore D. Detection and isolation of circulating exosomes and microvesicles for cancer monitoring and diagnostics using micro-/nano-based devices. Analyst 2015.

Marago OM, Jones PH, Gucciardi PG, Volpe G, Ferrari AC. Optical trapping and manipulation of nanostructures. Nat Nanotechnol 2013, 8:807-819.

Pang $\mathrm{Y}$, Song H, Kim JH, Hou X, Cheng W. Optical trapping of individual human immunodeficiency viruses in culture fluid reveals heterogeneity with single-molecule resolution. Nat Nanotechnol 2014, 9:624-630.

Yurt A, Daaboul GG, Connor JH, Goldberg BB, Unlu MS. Single nanoparticle detectors for biological applications. Nanoscale 2012, 4:715-726.

Related Articles

\begin{tabular}{|c|l|}
\hline DOI & \multicolumn{1}{|c|}{ Article title } \\
\hline $10.1038 /$ nnano.2014.140 & $\begin{array}{l}\text { Optical trapping of individual human immunodeficiency viruses in } \\
\text { culture fluid reveals heterogeneity with single-molecule resolution }\end{array}$ \\
\hline & \\
\hline & \\
\hline
\end{tabular}

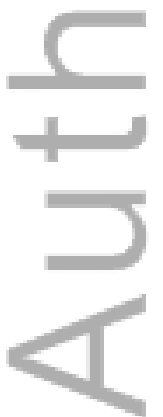

This article is protected by copyright. All rights reserved. 

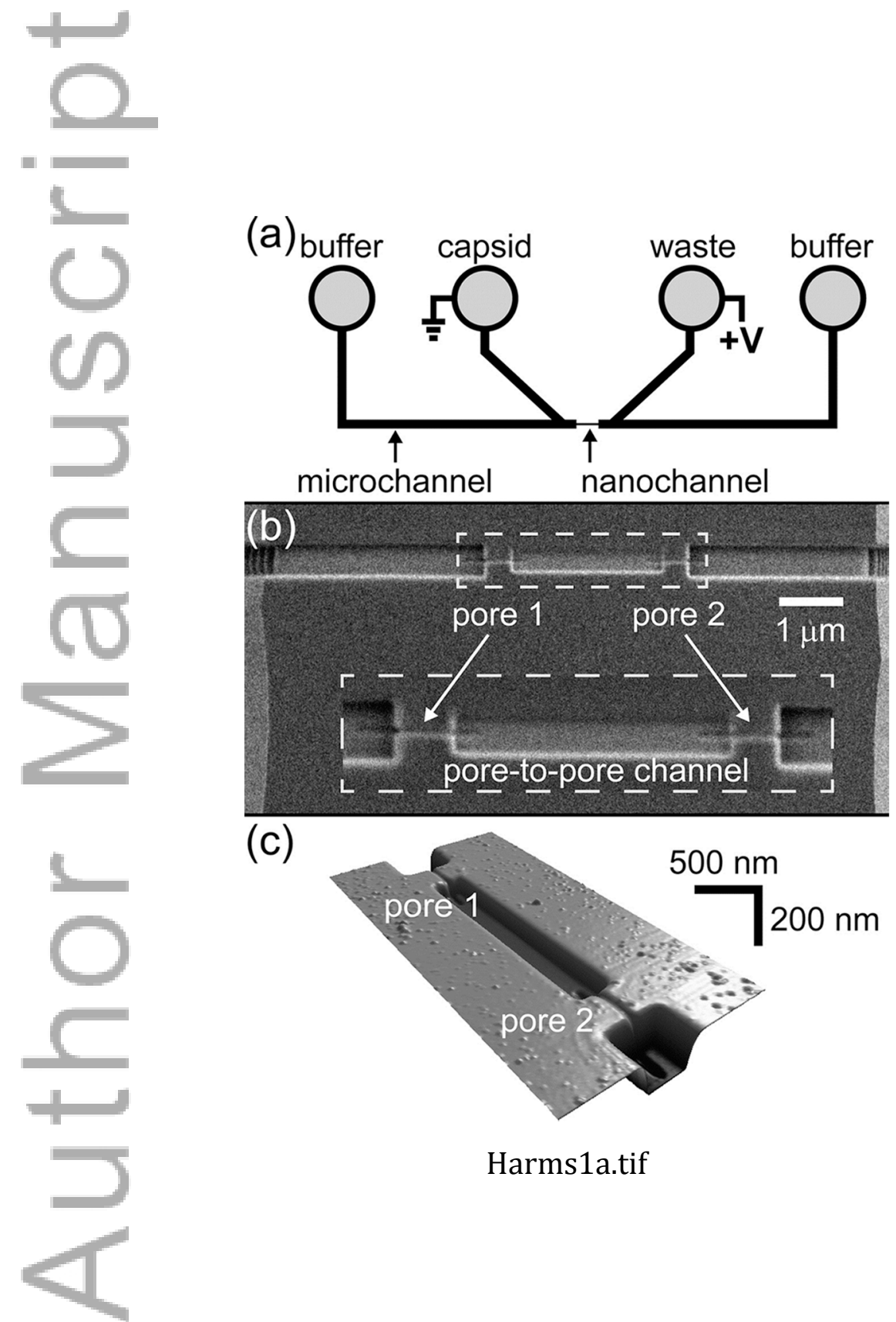

(c)

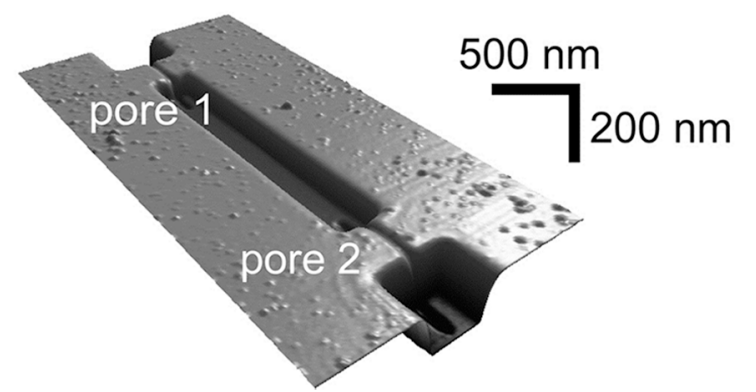

Harms1a.tif

This article is protected by copyright. All rights reserved. 


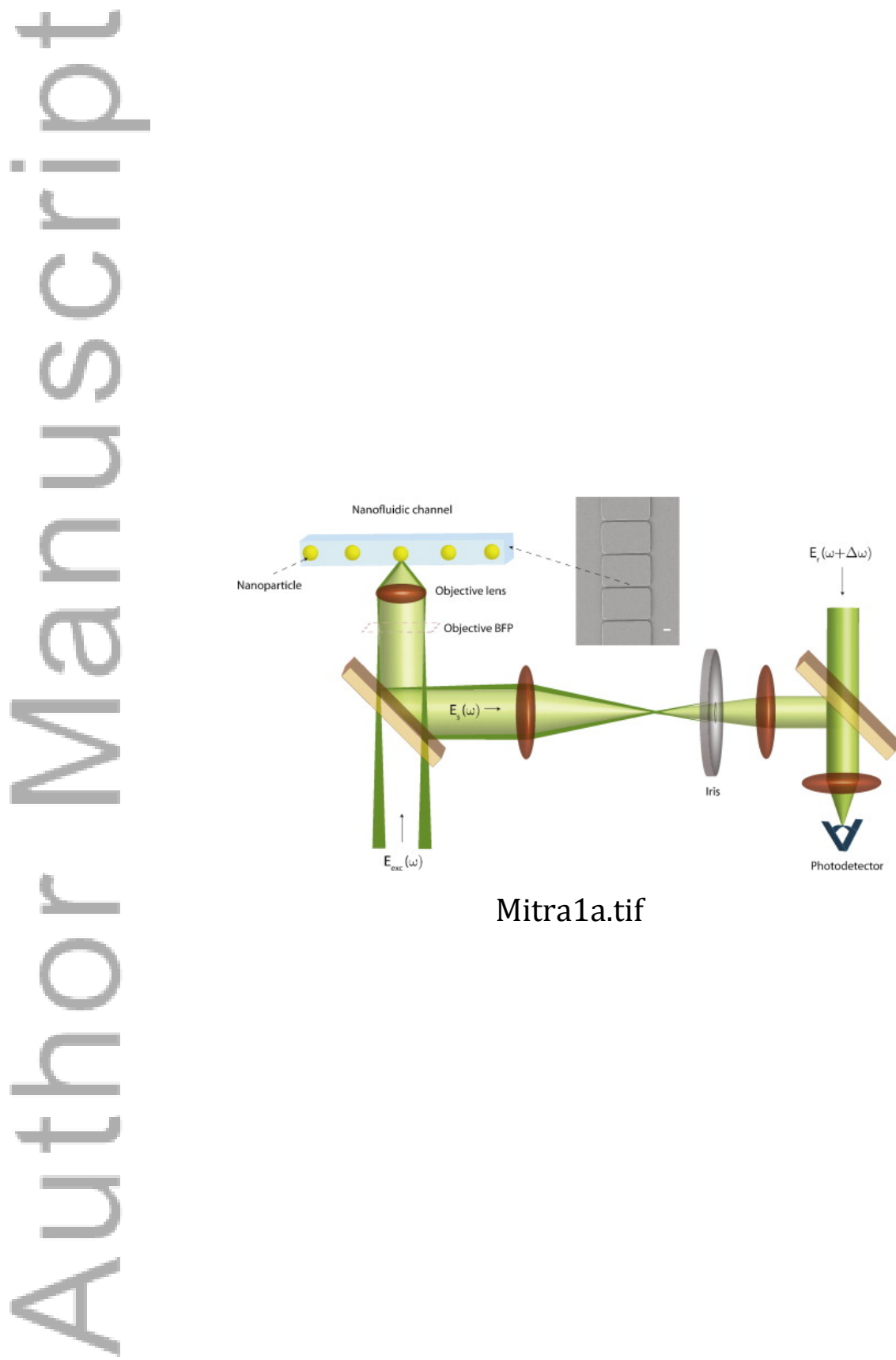

This article is protected by copyright. All rights reserved. 

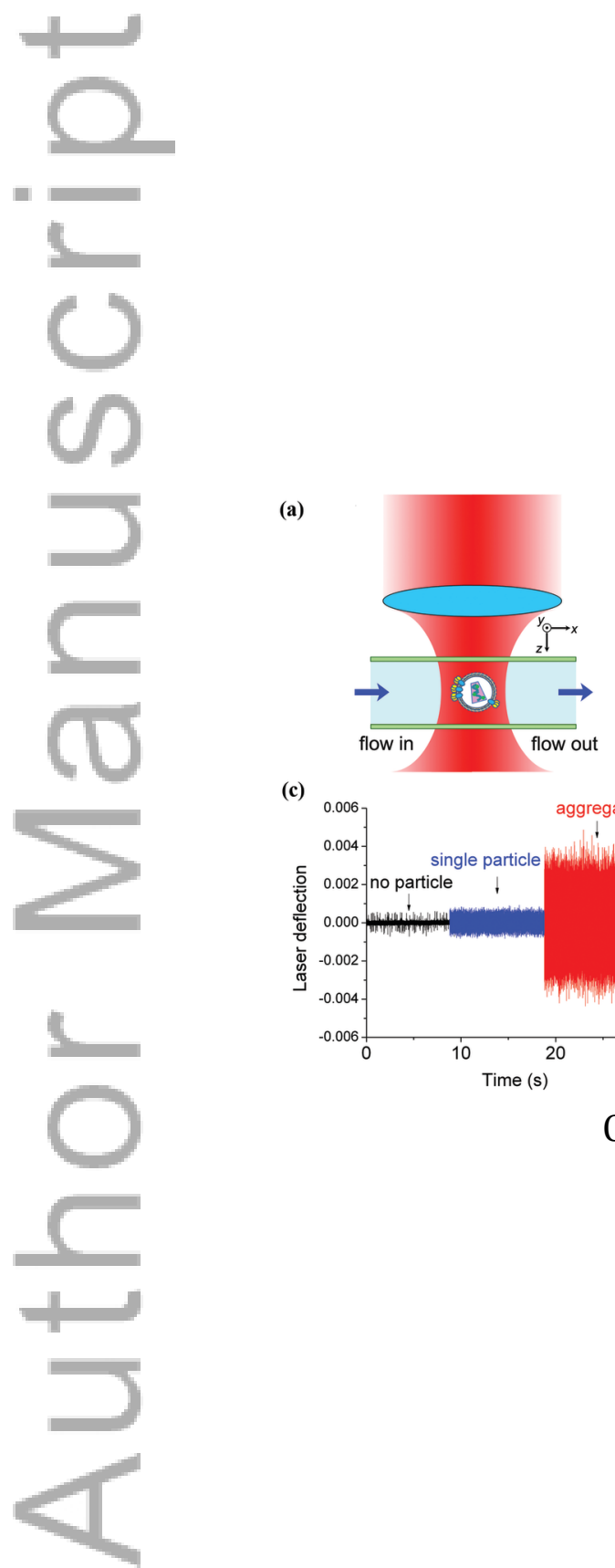

(b)
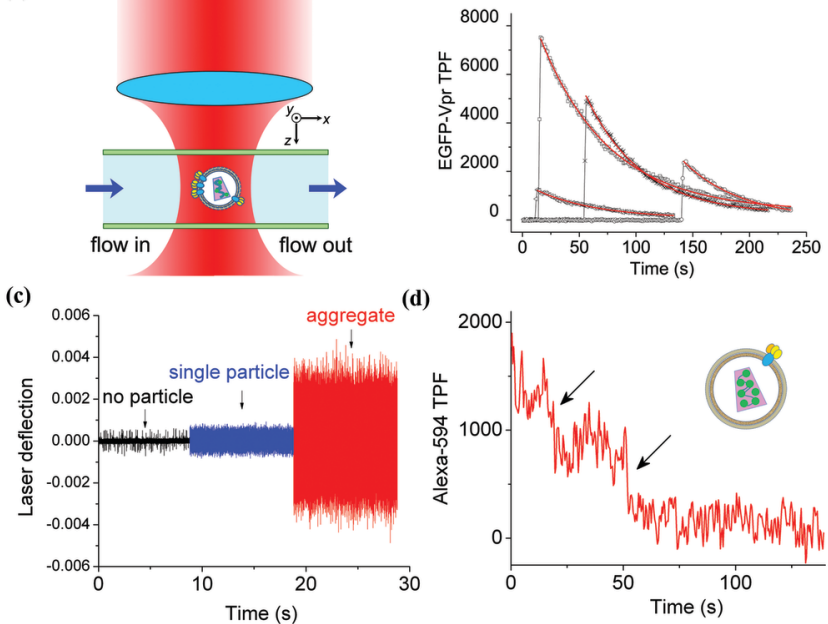

(d)

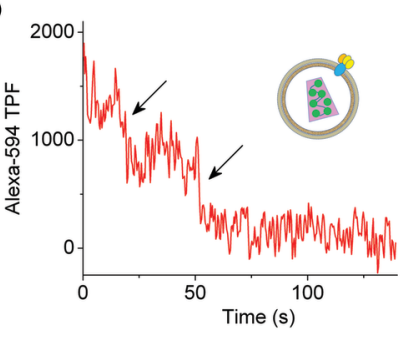

OTV.tif

This article is protected by copyright. All rights reserved. 

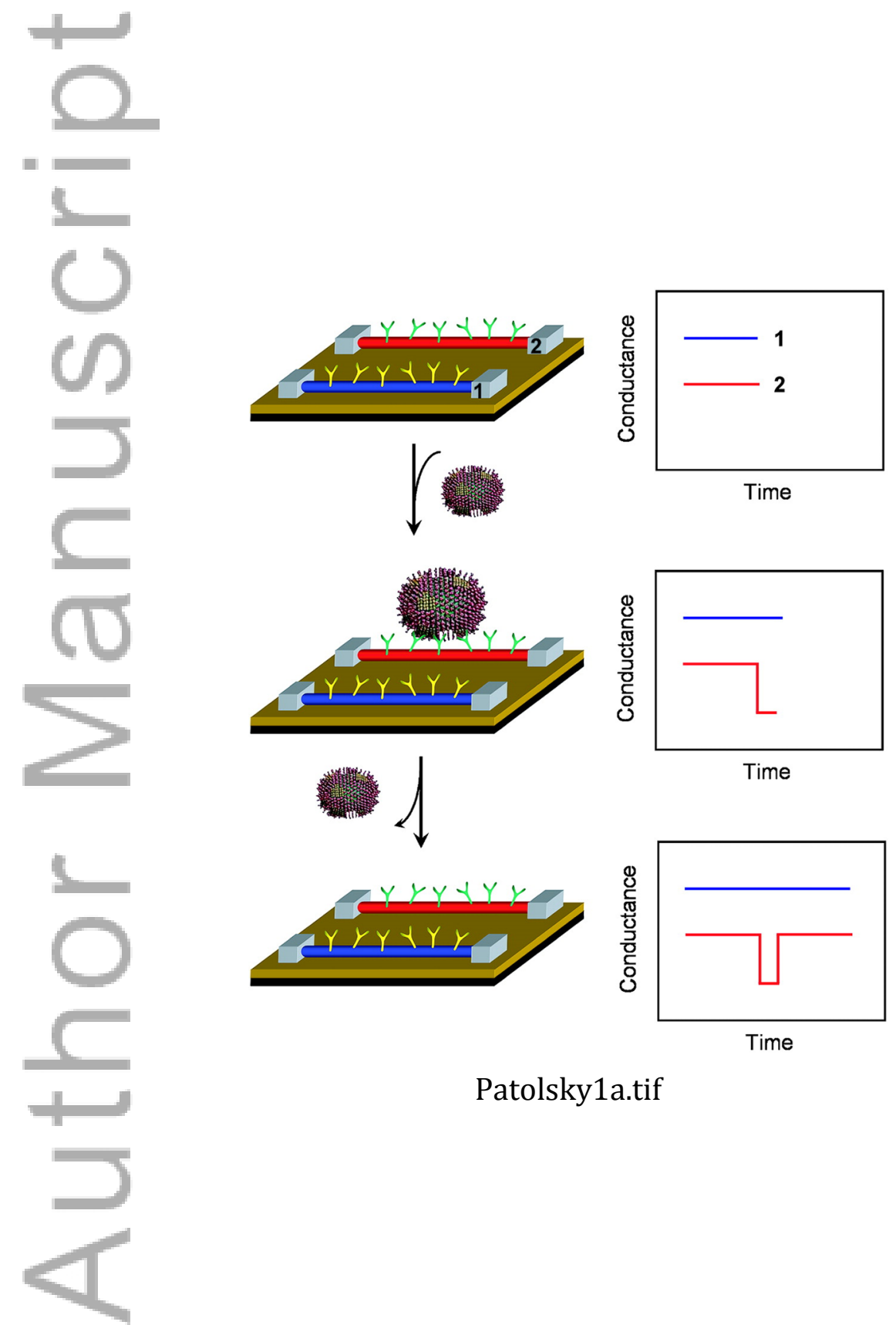

Patolsky1a.tif

This article is protected by copyright. All rights reserved. 

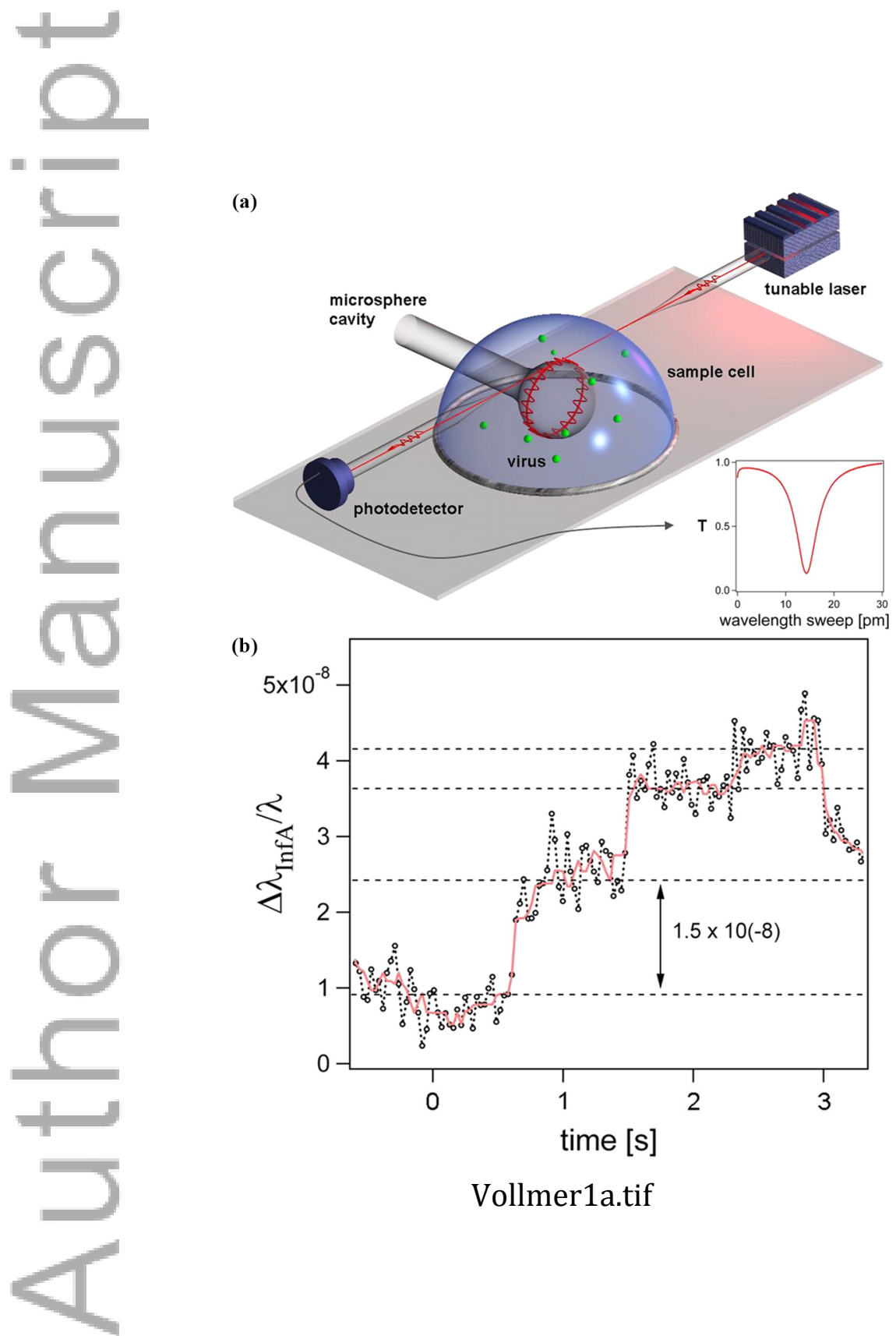

Vollmer1a.tif

This article is protected by copyright. All rights reserved. 

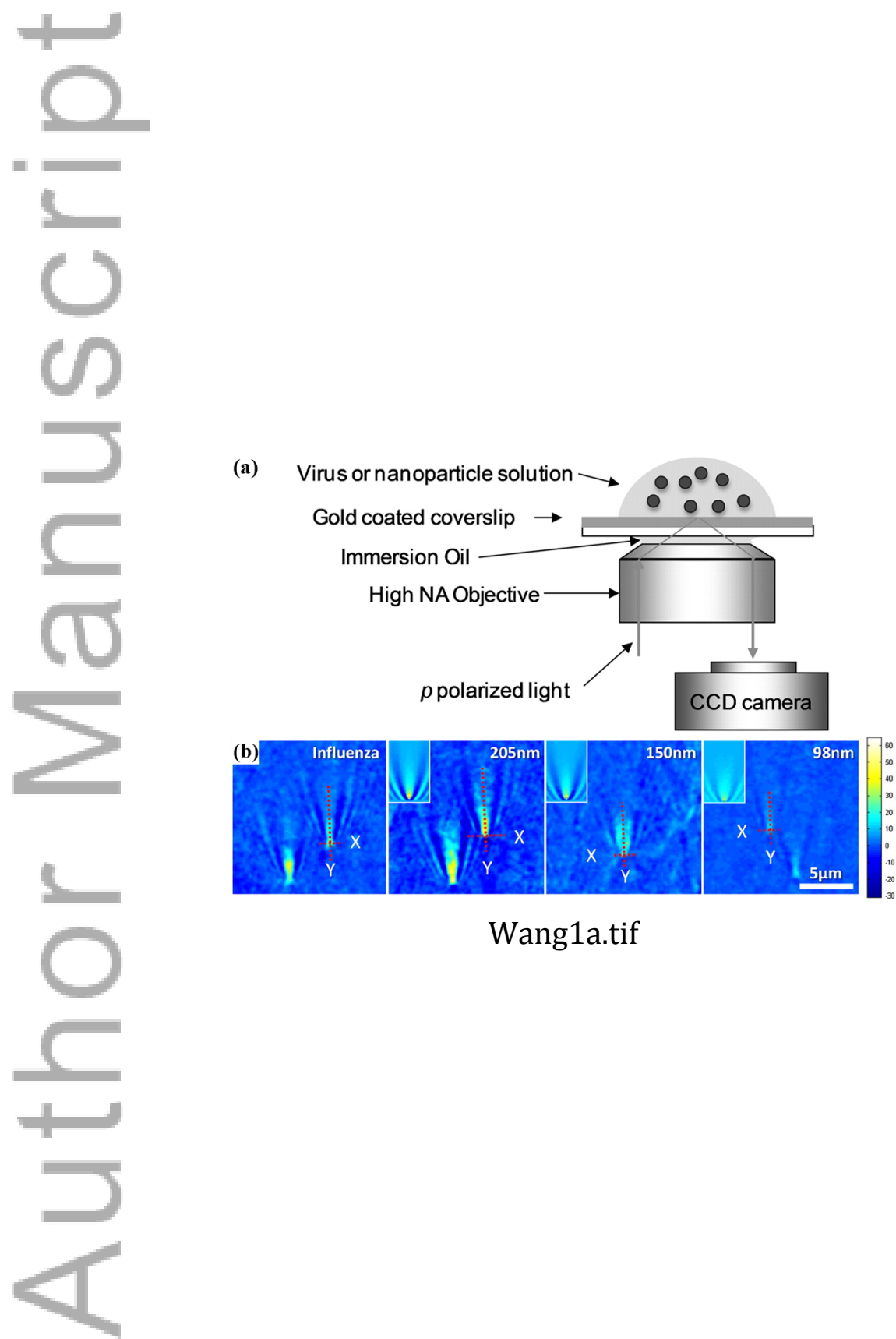

Wang1a.tif

This article is protected by copyright. All rights reserved. 